\title{
An algorithm for the numerical solution of two-sided space-fractional partial differential equations
}

\author{
Neville J. Ford, Kamal Pal, Yubin Yan ${ }^{1}$
}

\begin{abstract}
We introduce an algorithm for solving two-sided space-fractional partial differential equations. The spacefractional derivatives we consider here are left-handed and right-handed Riemann-Liouville fractional derivatives which are expressed by using Hadamard finite-part integrals. We approximate the Hadamard finite-part integrals by using piecewise quadratic interpolation polynomials and obtain a numerical approximation of the space-fractional derivative with convergence order $O\left(\Delta x^{3-\alpha}\right), 1<\alpha<2$. A shifted implicit finite difference method is applied for solving the two-sided space-fractional partial differential equation and we prove that the order of convergence of the finite difference method is $O\left(\Delta t+\Delta x^{\min (3-\alpha, \beta)}\right), 1<\alpha<2, \beta>0$, where $\Delta t, \Delta x$ denote the time and space step sizes, respectively. Numerical examples, where the solutions have varying degrees of smoothness are presented and compared with the exact analytical solution to compare the practical performance of the method with the theoretical order of convergence.
\end{abstract}

Key words:

finite difference methods, error estimates, Riemman-Liouville derivative, space-fractional partial differential equations

AMS Subject Classification: 65M12; 65M06; 65M70;35S10

\section{Introduction}

Consider the following two-sided space-fractional partial differential equation, with $1<\alpha<2, t>0$,

$$
\begin{aligned}
& u_{t}(t, x)=C_{+}(t, x){ }_{0}^{R} D_{x}^{\alpha} u(t, x),+C_{-}(t, x){ }_{x}^{R} D_{1}^{\alpha} u(t, x)+f(t, x), \quad 0<x<1, \\
& u(t, 0)=\varphi_{1}(t), \quad u(t, 1)=\varphi_{2}(t), \\
& u(0, x)=u_{0}(x), \quad 0<x<1 .
\end{aligned}
$$

Here the function $f(t, x)$ is a source/sink term. The functions $C_{+}(t, x) \geq 0$ and $C_{-}(t, x) \geq 0$ may be interpreted as transport related coefficients. The addition of a classical advective term $-\nu(t, x) \frac{\partial u(t, x)}{\partial x}$ in (1) does not impact the analysis performed in this paper, and has been omitted to simplify the notation [24]. The left-handed fractional derivative ${ }_{0}^{R} D_{x}^{\alpha} f(x)$ and right-handed fractional derivative ${ }_{x}^{R} D_{1}^{\alpha} f(x)$ in (1) are Riemann-Liouville fractional derivatives of order $\alpha$ defined by, with $1<\alpha<2$,

$$
{ }_{0}^{R} D_{x}^{\alpha} f(x)=\frac{1}{\Gamma(2-\alpha)} \frac{d^{2}}{d x^{2}} \int_{0}^{x}(x-\xi)^{1-\alpha} f(\xi) d \xi,
$$

and

$$
{ }_{x}^{R} D_{1}^{\alpha} f(x)=\frac{1}{\Gamma(2-\alpha)} \frac{d^{2}}{d x^{2}} \int_{x}^{1}(\xi-x)^{1-\alpha} f(\xi) d \xi
$$

Email addresses: njford@chester.ac.uk (Neville J. Ford), kamal_pal08@yahoo.co.uk (Kamal Pal), y.yan@chester.ac.uk (Yubin Yan) 
There are several ways to approximate the Riemann- Liouville fractional derivative. Let $0=x_{0}<x_{1}<$ $\cdots<x_{j}<\cdots<x_{M}=1$ be a partition of $[0,1]$ and $\Delta x$ the stepsize. Based on the definition of the Grünwald-Letnikov derivative, one can approximate the left-handed and right-handed Riemann-Liouville fractional derivatives by, [24],

$$
{ }_{0}^{R} D_{x}^{\alpha} f\left(x_{j}\right)=\Delta x^{-\alpha} \sum_{k=0}^{j} w_{k}^{(\alpha)} f\left(x_{j-k}\right)+O(\Delta x)
$$

and

$$
{ }_{x}^{R} D_{1}^{\alpha} f\left(x_{j}\right)=\Delta x^{-\alpha} \sum_{k=0}^{M-j} w_{k}^{(\alpha)} f\left(x_{j+k}\right)+O(\Delta x),
$$

where $w_{k}^{(\alpha)}$ are some weights and the order of convergence in (6) or (7) is $O(\Delta x)$ for any $\alpha>0$. Lubich [20] obtained approximations of order $2-6$ in the form of (6), where the coefficients $w_{k}^{(\alpha)}$ are just the coefficients of the Taylor series expansions of some generating functions $w_{l}^{(\alpha)}(z), l=2,3,4,5,6$. However, there is a need to have special starting weights to recover the order of these methods because the solution may not be smooth. The $L 2$ scheme and its modification $L 2 C$ scheme are introduced in Oldham and Spanier [27], Lynch, et al. [21] as follows. Note that, with $1<\alpha<2$,

$$
\begin{array}{r}
{ }_{0}^{R} D_{x}^{\alpha} f\left(x_{j}\right)=\frac{f\left(x_{0}\right)\left(x_{j}-x_{0}\right)^{-\alpha}}{\Gamma(1-\alpha)}+\frac{f^{\prime}\left(x_{0}\right)\left(x_{j}-x_{0}\right)^{1-\alpha}}{\Gamma(2-\alpha)} \\
+\frac{1}{2-\alpha} \sum_{l=0}^{j-1} \int_{x_{l}}^{x_{l+1}} s^{1-\alpha} f^{\prime \prime}\left(x_{j}-s\right) d s .
\end{array}
$$

On each interval $\left[x_{l}, x_{l+1}\right], f^{\prime \prime}\left(x_{j}-s\right)$ is approximated by $\left(f\left(x_{j}-x_{l}\right)-2 f\left(x_{j}-x_{l+1}\right)+f\left(x_{j}-x_{l+2}\right)\right) / \Delta x^{2}$, then the so-called $L 2$ scheme is obtained and the convergence order is $O(\Delta x)$. Similarly one can obtain $L 2 C$ scheme. Diethelm [5] [6] expressed the Riemann-Liouville fractional derivative by the equivalent Hadamard finite-part integral and approximated this Hadamard finite-part integral by using piecewise linear interpolation polynomials. More precisely, Diethelm [6] obtained, with $1<\alpha<2$,

$$
\begin{aligned}
{ }_{0}^{R} D_{x}^{\alpha} f\left(x_{j}\right) & =\frac{1}{\Gamma(2-\alpha)} \int_{0}^{x_{j}}\left(x_{j}-\xi\right)^{-\alpha} f^{\prime}(x) d x=\frac{1}{\Gamma(-\alpha)} \oint_{0}^{x_{j}}\left(x_{j}-\xi\right)^{-\alpha-1} f(\xi) d \xi \\
& =\Delta x^{-\alpha} \sum_{k=0}^{j} w_{k, j} f\left(x_{j-k}\right)+O\left(\Delta x^{2-\alpha}\right),
\end{aligned}
$$

where $\oint_{0}^{x_{j}}$ denotes the Hadamard finite-part integral and $w_{k, j}$ are some weights.

Odibat [26] introduced a computational algorithm for approximating the Caputo fractional derivative and the convergence order is $O\left(\Delta x^{2}\right)$, see also Sousa [30]. The idea is as follows. Note that, with $1<\alpha<2$,

$$
\begin{aligned}
{ }_{0}^{C} D_{x}^{\alpha} f\left(x_{j}\right) & =\frac{1}{\Gamma(2-\alpha)} \int_{0}^{x_{j}}\left(x_{j}-\xi\right)^{1-\alpha} f^{\prime \prime}(\xi) d \xi \\
& =\frac{1}{\Gamma(2-\alpha)} \sum_{l=0}^{j-1} \int_{x_{l}}^{x_{l+1}}\left(x_{j}-\xi\right)^{1-\alpha} f^{\prime \prime}(\xi) d \xi
\end{aligned}
$$

On each subinterval $\left[x_{l}, x_{l+1}\right]$, one approximates the integral by using linear interpolation polynomial $P_{1}(\xi)=$ $\frac{\xi-x_{l+1}}{x_{l}-x_{l+1}} f^{\prime \prime}\left(x_{l}\right)+\frac{\xi-x_{l}}{x_{l+1}-x_{l}} f^{\prime \prime}\left(x_{l+1}\right)$ and obtain, with some weights $\bar{w}_{k, j}, k=0,1,2, \ldots, j$,

$$
{ }_{0}^{C} D_{x}^{\alpha} f\left(x_{j}\right) \approx \frac{1}{\Gamma(2-\alpha)} \sum_{l=0}^{j-1} \int_{x_{l}}^{x_{l+1}}\left(x_{j}-\xi\right)^{1-\alpha} P_{1}(\xi) d \xi=\Delta x^{2-\alpha} \sum_{k=0}^{j} \bar{w}_{k, j} f^{\prime \prime}\left(x_{k}\right) .
$$


Further, Odibat [26] approximated $f^{\prime \prime}\left(x_{k}\right)$ by $\frac{f\left(x_{k+1}\right)-2 f\left(x_{k}\right)+f\left(x_{k-1}\right)}{\Delta x^{2}}$ and obtained a second order approximation scheme to ${ }_{0}^{C} D_{x}^{\alpha} f\left(x_{j}\right)$. More recently, Dimitrov [8] obtained a second and third order approximations of Caputo derivative by the Grünwald and shifted Grünwald formulae with weighted averages.

There is considerable interest in developing various numerical methods for solving space-fractional partial differential equations in literature: the finite difference methods [2], [13], [14] - [15], [18] - [19], [21] - [25], [31] - [35], the finite element methods, [3] - [4], [9] - [12] and the spectral methods [16]-[17]. By using shifted Grünwald -Letnikov formulae (6) and (7), Meerschaert and Tadjeran [24] introduced a finite difference method for solving two-sided space-fractional partial differential equations (1)- (3) and proved that the convergence order of spatial discretisation is $O(\Delta x)$. Meerschaert and Tadjeran [23] also considered the finite difference method for solving 1D fractional advection-dispersion equation, with $1<\alpha<2$,

$$
\frac{\partial u(t, x)}{\partial t}=-\nu(x) \frac{\partial u(t, x)}{\partial x}+d(x) \frac{\partial^{\alpha} u(t, x)}{\partial x^{\alpha}}+f(t, x),
$$

by using shifted Grünwald-Letnikov formula on a finite domain and proved that the convergence order of spatial discretisation is $O(\Delta x)$. Tadjeran, Meerschaert and Scheffler [31] and Tadjeran and Meerschaert [32] applied shifted Grünwald-Letnikov formula and extrapolation techniques to fractional diffusion equation in $1 \mathrm{D}$ and $2 \mathrm{D}$ and obtained a second-order accurate finite difference method. Liu et al. [19] transformed the fractional advection-dispersion equation into a system of ordinary differential equations, which was then solved using backward difference formulae. Podlubny [28] and Podlubny et al. [29] developed a matrix approach to discretise fractional diffusion equations with various combinations of time-space-fractional derivatives.

In this paper, we will use the idea in Diethelm [6] to define a finite difference method for solving (1)(3), see also our recent works in [13], [14]. We first express the fractional derivative by using the Hadamard finite-part integral, i.e., with $1<\alpha<2$,

$$
{ }_{0}^{R} D_{x}^{\alpha} f(x)=\frac{1}{\Gamma(2-\alpha)} \frac{d^{2}}{d x^{2}} \int_{0}^{x}(x-\xi)^{1-\alpha} f(\xi) d \xi=\frac{1}{\Gamma(-\alpha)} \oint_{0}^{x}(x-\xi)^{-\alpha-1} f(\xi) d \xi .
$$

Then we approximate $f(\xi)$ by using the piecewise quadratic interpolation polynomials and obtain an approximation scheme of Riemann-Liouville fractional derivative. Similarly we can approximate the right-handed Riemann-Liouville fractional derivative ${ }_{x}^{R} D_{1}^{\alpha} f(x)$. Based on these approximation schemes, we define a shifted finite difference method for solving (1)-(3). We prove that the convergence order of the numerical method is $O\left(\Delta t+\Delta x^{\min (3-\alpha, \beta)}\right), 1<\alpha<2, \beta>0$.

The paper is organised as follows. In Section 2, we consider the implicit shifted finite difference method for solving (1)-(3) where the Hadamard integral of the space-fractional derivative is approximated by using piecewise quadratic interpolation polynomials. In Section 3, we give three numerical examples. The numerical experiments are consistent with the theoretical results.

Remark 1.1. In this paper we are studying a relatively low order scheme, and we consider (through example) how the method will perform when the convergence smoothness conditions on the solution are violated. We observe in the example that there is some loss of order even in this low order scheme but that the scheme is still more effective than competitor schemes.. We also consider how the method will perform under the nonhomogeneous Dirichlet boundary conditions. Again, we observe in the example that there is a loss of order even in this lower order scheme, but once again its performance is better than competitors.

\section{Numerical methods}

In this section, we will introduce a finite difference method for solving (1)-(3) by using the idea in Diethelm [5]. For simplicity, we assume $C_{+}(t, x)=C_{-}(t, x)=1$ and $\varphi_{1}(t)=\varphi_{2}(t)=0$. Recall that the Riemann-Liouville fractional derivative has the form, with $1<\alpha<2$,

$$
{ }_{0}^{R} D_{x}^{\alpha} f(x)=\frac{1}{\Gamma(-\alpha)} \oint_{0}^{x}(x-\xi)^{-1-\alpha} f(\xi) d \xi,
$$


where $\oint_{0}^{x}(x-\xi)^{-1-\alpha} f(\xi) d \xi$ denotes the Hadamard finite-part integral [5].

Let $m$ be a fixed positive integer and $M=2 m$. Let $0=x_{0}<x_{1}<x_{2}<\cdots<x_{2 j}<x_{2 j+1}<\cdots<$ $x_{2 m}=1$ be a partition of $[0,1]$ and $\Delta x$ the step size.

At the nodes $x_{2 j}=\frac{2 j}{2 m}, j=1,2, \ldots, m$, we have

$$
{ }_{0}^{R} D_{x}^{\alpha} f\left(x_{2 j}\right)=\frac{1}{\Gamma(-\alpha)} \oint_{0}^{x_{2 j}}\left(x_{2 j}-\xi\right)^{-1-\alpha} f(\xi) d \xi=\frac{x_{2 j}^{-\alpha}}{\Gamma(-\alpha)} \oint_{0}^{1} w^{-1-\alpha} f\left(x_{2 j}-x_{2 j} w\right) d w .
$$

For every $j$, we replace $g(w)=f\left(x_{2 j}-x_{2 j} w\right)$ in the integral in (9) by piecewise quadratic interpolation polynomials with the equispaced nodes $0, \frac{1}{2 j}, \frac{2}{2 j}, \ldots, \frac{2 j}{2 j}$. We then have

$$
\oint_{0}^{1} w^{-1-\alpha} g(w) d w=\oint_{0}^{1} w^{-1-\alpha} P_{2}(w) d w+R_{2 j}(g),
$$

where $P_{2}(w)$ is the piecewise quadratic interpolation polynomial of $g(w)$ defined on the equispaced nodes $0, \frac{1}{2 j}, \frac{2}{2 j}, \ldots, \frac{2 j}{2 j}$ and $R_{2 j}(g)$ is the remainder term.

At the node $x_{2 j+1}=\frac{2 j+1}{2 m}, j=1,2, \ldots, m-1$ we have

$$
\begin{aligned}
{ }_{0}^{R} D_{x}^{\alpha} f\left(x_{2 j+1}\right) & =\frac{1}{\Gamma(-\alpha)} \oint_{0}^{x_{2 j+1}}\left(x_{2 j+1}-\xi\right)^{-1-\alpha} f(\xi) d \xi \\
& =\frac{1}{\Gamma(-\alpha)} \int_{0}^{x_{1}}\left(x_{2 j+1}-\xi\right)^{-1-\alpha} f(\xi) d \tau \\
& +\frac{x_{2 j+1}^{-\alpha}}{\Gamma(-\alpha)} \oint_{0}^{\frac{2 j}{2 j+1}} w^{-1-\alpha} f\left(x_{2 j+1}-x_{2 j+1} w\right) d w .
\end{aligned}
$$

For every $j, j=1,2, \ldots, m-1$, we replace $g(w)=f\left(x_{2 j+1}-x_{2 j+1} w\right)$ by a piecewise quadratic interpolation polynomial with the equispaced nodes $0, \frac{1}{2 j+1}, \frac{2}{2 j+1}, \ldots, \frac{2 j}{2 j+1}$ and obtain

$$
\oint_{0}^{\frac{2 j}{2 j+1}} w^{-1-\alpha} g(w) d w=\oint_{0}^{\frac{2 j}{2 j+1}} w^{-1-\alpha} Q_{2}(w) d w+R_{2 j+1}(g),
$$

where $Q_{2}(w)$ is the piecewise quadratic interpolation polynomial of $g(w)$ defined on the nodes $0, \frac{1}{2 j+1}, \frac{2}{2 j+1}, \ldots, \frac{2 j}{2 j+1}$ and $R_{2 j+1}(g)$ is the remainder term.

We have, see [34]

Lemma 2.1. Let $1<\alpha<2$ and let $M=2 m$ where $m$ is a fixed positive integer. Let $0=x_{0}<x_{1}<x_{2}<$ $\cdots<x_{2 j}<x_{2 j+1}<\cdots<x_{M}=1$ be a partition of $[0,1]$. Assume that $f \in C^{3}[0,1]$ is a sufficiently smooth function. Then we have, with $j=1,2, \ldots, m$,

$$
\begin{aligned}
\left.{ }_{0}^{R} D_{x}^{\alpha} f(x)\right|_{x=x_{2 j}} & =\frac{x_{2 j}^{-\alpha}}{\Gamma(-\alpha)}\left(\sum_{l=0}^{2 j} \alpha_{l, 2 j} f\left(x_{2 j-l}\right)+R_{2 j}(f)\right) \\
& =\Delta x^{-\alpha} \sum_{l=0}^{2 j} w_{l, 2 j} f\left(x_{2 j-l}\right)+\frac{x_{2 j}^{-\alpha}}{\Gamma(-\alpha)} R_{2 j}(f),
\end{aligned}
$$

and, with $j=1,2, \ldots, m-1$,

$$
\begin{aligned}
& \left.{ }_{0}^{R} D_{x}^{\alpha} f(x)\right|_{x=x_{2 j+1}}=\frac{1}{\Gamma(-\alpha)} \int_{0}^{x_{1}}\left(x_{2 j+1}-\xi\right)^{-1-\alpha} f(\xi) d \xi \\
& \quad+\frac{x_{2 j+1}^{-\alpha}}{\Gamma(-\alpha)}\left(\sum_{l=0}^{2 j} \alpha_{l, 2 j+1} f\left(x_{2 j+1-l}\right)+R_{2 j+1}(f)\right) \\
& =\frac{1}{\Gamma(-\alpha)} \int_{0}^{x_{1}}\left(x_{2 j+1}-\xi\right)^{-1-\alpha} f(\xi) d \xi+\Delta x^{-\alpha} \sum_{l=0}^{2 j} w_{l, 2 j+1} f\left(x_{2 j+1-l}\right)+\frac{x_{2 j+1}^{-\alpha}}{\Gamma(-\alpha)} R_{2 j+1}(f),
\end{aligned}
$$


where

$$
\begin{aligned}
&(-\alpha)(-\alpha+1)(-\alpha+2)(2 j)^{-\alpha} \alpha_{l, 2 j} \\
&= \begin{cases}2^{-\alpha}(\alpha+2), & \text { for } l=0, \\
(-\alpha) 2^{2-\alpha}, & \text { for } l=1, \\
(-\alpha)\left(-2^{-\alpha} \alpha\right)+\frac{1}{2} F_{0}(2), & \text { for } l=2, \\
-F_{1}(k), & \text { for } l=2 k-1, \quad k=2,3, \ldots, j, \\
\frac{1}{2}\left(F_{2}(k)+F_{0}(k+1)\right), & \text { for } l=2 k, \quad k=2,3, \ldots, j-1, \\
\frac{1}{2} F_{2}(j), & \text { for } l=2 j,\end{cases} \\
& F_{0}(k)=(2 k-1)(2 k)\left((2 k)^{-\alpha}-(2(k-1))^{-\alpha}\right)(-\alpha+1)(-\alpha+2)
\end{aligned}
$$

Further we have, with $l=0,1,2, \ldots, 2 j$,

$$
\Gamma(3-\alpha) w_{l, 2 j}=(-\alpha)(-\alpha+1)(-\alpha+2)(2 j)^{-\alpha} \alpha_{l, 2 j},
$$

and

$$
\alpha_{l, 2 j+1}=\alpha_{l, 2 j}, \quad w_{l, 2 j+1}=w_{l, 2 j} .
$$

The remainder term $R_{l}(f)$ satisfies, for every $f \in C^{3}(0,1)$,

$$
\left|R_{l}(f)\right| \leq C \Delta x^{3-\alpha}\left\|f^{\prime \prime \prime}\right\|_{\infty}, l=2,3,4, \ldots, M, \text { with } M=2 m .
$$

We can consider in a similar way the approximation of the right-handed fractional derivative ${ }_{x}^{R} D_{1}^{\alpha} f(x)$ at $x=x_{l}, l=0,1,2, \ldots, 2 m-2$. Using the same argument as for the approximation of ${ }_{0}^{R} D_{x}^{\alpha} f(x)$ at $x=x_{l}$, we can show that, with $j=0,1,2, \ldots, m-1$,

$$
\left.{ }_{x}^{R} D_{1}^{\alpha} f(x)\right|_{x=x_{2 j}}=\Delta x^{-\alpha} \sum_{l=0}^{M-2 j} w_{l, M-2 j} f\left(x_{2 j+l}\right)+\frac{x_{2 j}^{-\alpha}}{\Gamma(-\alpha)} R_{2 j}(f),
$$

and, with $j=0,1,2, \ldots, m-2$,

$$
\begin{aligned}
& \left.{ }_{x}^{R} D_{1}^{\alpha} f(x)\right|_{x=x_{2 j+1}}=\frac{1}{\Gamma(-\alpha)} \int_{x_{M-1}}^{x_{M}}\left(\xi-x_{2 j+1}\right)^{-1-\alpha} f(\xi) d \xi \\
& +\Delta x^{-\alpha} \sum_{l=0}^{M-(2 j+1)-1} w_{l, M-(2 j+1)} f\left(x_{2 j+1+l}\right)+\frac{x_{2 j+1}^{-\alpha}}{\Gamma(-\alpha)} R_{2 j+1}(f)
\end{aligned}
$$


Let $0=t_{0}<t_{1}<t_{2}<\cdots<t_{n}<\ldots$ be the time partition and $\Delta t$ the time step size. At the nodes $x_{2 j}=\frac{2 j}{2 m}, j=1,2, \ldots, m-1$, we have, by $(1)$,

$$
u_{t}\left(t_{n+1}, x_{2 j}\right)-\left({ }_{0}^{R} D_{x}^{\alpha} u\left(t_{n+1}, x_{2 j+1}\right)+{ }_{x}^{R} D_{1}^{\alpha} u\left(t_{n+1}, x_{2 j-1}\right)\right)=f_{2 j}^{n+1}+\sigma_{2 j}^{n+1},
$$

and at the nodes $x_{2 j+1}=\frac{2 j+1}{2 m}, j=1,2, \ldots, m-1$,

$$
u_{t}\left(t_{n+1}, x_{2 j+1}\right)-\left({ }_{0}^{R} D_{x}^{\alpha} u\left(t_{n+1}, x_{2 j+2}\right)+{ }_{x}^{R} D_{1}^{\alpha} u\left(t_{n+1}, x_{2 j}\right)\right)=f_{2 j+1}^{n+1}+\sigma_{2 j+1}^{n+1},
$$

where

$$
\begin{gathered}
\sigma_{2 j}^{n+1}=-\left({ }_{0}^{R} D_{x}^{\alpha} u\left(t_{n+1}, x_{2 j+1}\right)-{ }_{0}^{R} D_{x}^{\alpha} u\left(t_{n+1}, x_{2 j}\right)\right) \\
-\left({ }_{x}^{R} D_{1}^{\alpha} u\left(t_{n+1}, x_{2 j-1}\right)-{ }_{x}^{R} D_{1}^{\alpha} u\left(t_{n+1}, x_{2 j}\right)\right), \\
\sigma_{2 j+1}^{n+1}=-\left({ }_{0}^{R} D_{x}^{\alpha} u\left(t_{n+1}, x_{2 j+2}\right)-{ }_{0}^{R} D_{x}^{\alpha} u\left(t_{n+1}, x_{2 j+1}\right)\right) \\
-\left({ }_{x}^{R} D_{1}^{\alpha} u\left(t_{n+1}, x_{2 j}\right)-{ }_{x}^{R} D_{1}^{\alpha} u\left(t_{n+1}, x_{2 j+1}\right)\right) .
\end{gathered}
$$

Discretising $u_{t}\left(t_{n+1}, x_{l}\right)$ by using backward Euler method and discretising ${ }_{0}^{R} D_{x}^{\alpha} u\left(t_{n+1}, x_{l}\right)$ and ${ }_{x}^{R} D_{1}^{\alpha} u\left(t_{n+1}, x_{l}\right)$ by using (13) - (14) and (21) - (22), respectively, we obtain, with $u_{j}^{n}=u\left(t_{n}, x_{j}\right), f_{j}^{n}=f\left(t_{n}, x_{j}\right)$

$$
\begin{aligned}
& \Delta t^{-1}\left(u_{2 j}^{n+1}-u_{2 j}^{n}\right)=\Delta x^{-\alpha}\left(\sum_{k=0}^{2 j} w_{k, 2 j+1} u_{2 j+1-k}^{n+1}+\sum_{k=0}^{M-(2 j-1)-1} w_{k, M-(2 j-1)} u_{2 j-1+k}^{n+1}\right) \\
& \quad+f_{2 j}^{n+1}+S_{2 j}^{n+1}+\sigma_{2 j}^{n+1}+\tau_{2 j}^{n+1}, j=1,2, \ldots, m-1 \\
& \Delta t^{-1}\left(u_{2 j+1}^{n+1}-u_{2 j+1}^{n}\right)=\Delta x^{-\alpha}\left(\sum_{k=0}^{2 j+2} w_{k, 2 j+2} u_{2 j+2-k}^{n+1}+\sum_{k=0}^{M-2 j} w_{k, M-2 j} u_{2 j+k}^{n+1}\right) \\
& \quad+f_{2 j+1}^{n+1}+\sigma_{2 j+1}^{n+1}+\tau_{2 j+1}^{n+1}, j=0,1,2, \ldots, m-1,
\end{aligned}
$$

where the truncation errors $\tau_{l}^{n+1}=O\left(\Delta t+\Delta x^{3-\alpha}\right), l=1,2, \ldots, \dot{M}-1[5],[6]$ and

$$
S_{2 j}^{n+1}=\frac{1}{\Gamma(-\alpha)} \int_{0}^{x_{1}}\left(x_{2 j+1}-\xi\right)^{-1-\alpha} u\left(\xi, t_{n+1}\right) d \xi+\frac{1}{\Gamma(-\alpha)} \int_{x_{M-1}}^{x_{M}}\left(\xi-x_{2 j+1}\right)^{-1-\alpha} u\left(\xi, t_{n+1}\right) d \xi .
$$

Let $U_{2 j}^{n} \approx u\left(t_{n}, x_{2 j}\right)$ and $U_{2 j+1}^{n} \approx u\left(t_{n}, x_{2 j+1}\right)$ denote the approximate solutions of $u\left(t_{n}, x_{2 j}\right)$ and $u\left(t_{n}, x_{2 j+1}\right)$, respectively. We define the following implicit shifted numerical method for solving (1) - (3).

$$
\begin{aligned}
& \Delta t^{-1}\left(U_{2 j}^{n+1}-U_{2 j}^{n}\right)=\Delta x^{-\alpha}\left(\sum_{k=0}^{2 j} w_{k, 2 j+1} U_{2 j+1-k}^{n+1}+\sum_{k=0}^{M-(2 j-1)-1} w_{k, M-(2 j-1)} u_{2 j-1+k}^{n+1}\right) \\
& \quad+f_{2 j}^{n+1}+Q_{2 j}^{n+1}, j=1,2, \ldots, m-1, \\
& \Delta t^{-1}\left(U_{2 j+1}^{n+1}-U_{2 j+1}^{n}\right)=\Delta x^{-\alpha}\left(\sum_{k=0}^{2 j+2} w_{k, 2 j+2} U_{2 j+2-k}^{n+1}+\sum_{k=0}^{M-2 j} w_{k, M-2 j} U_{2 j+k}^{n+1}\right) \\
& \quad+f_{2 j+1}^{n+1}, j=0,1,2, \ldots, m-1,
\end{aligned}
$$

where $Q_{2 j}^{n+1}$ is defined below in (30). 
To approximate the integral $\frac{1}{\Gamma(-\alpha)} \int_{0}^{x_{1}}\left(x_{2 j+1}-\xi\right)^{-1-\alpha} u\left(t_{n+1}, \xi\right) d \xi$ in $(27)$, we denote $g(\xi)=u\left(t_{n+1}, \xi\right)$ and approximate $g(\xi)$ on $\left[0, x_{1}\right]$ by the following quadratic interpolation polynomials, [1],

$$
\begin{aligned}
P_{2}(\xi)= & \frac{\left(\xi-x_{\frac{1}{2}}\right)\left(\xi-x_{1}\right)}{\left(x_{0}-x_{\frac{1}{2}}\right)\left(x_{0}-x_{1}\right)} u\left(t_{n+1}, x_{0}\right)+\frac{\left(\xi-x_{0}\right)\left(\xi-x_{1}\right)}{\left(x_{\frac{1}{2}}-x_{0}\right)\left(x_{\frac{1}{2}}-x_{1}\right)} u\left(t_{n+1}, x_{\frac{1}{2}}\right) \\
& +\frac{\left(\xi-x_{0}\right)\left(\xi-x_{\frac{1}{2}}\right)}{\left(x_{1}-x_{0}\right)\left(x_{1}-x_{\frac{1}{2}}\right)} u\left(t_{n+1}, x_{1}\right) \quad \text { for } \xi \in\left[x_{0}, x_{1}\right],
\end{aligned}
$$

where

$$
u\left(t_{n+1}, \xi\right)-P_{2}(\xi)=R_{1}^{(1)}(\xi)=\frac{u^{\prime \prime \prime}\left(t_{n+1}, c_{1}\right)}{3 !}\left(\xi-x_{0}\right)\left(\xi-x_{\frac{1}{2}}\right)\left(\xi-x_{1}\right), c_{1} \in\left(0, x_{1}\right) .
$$

Further we approximate the value $u\left(t_{n+1}, x_{\frac{1}{2}}\right)$ by

$$
u\left(t_{n+1}, x_{\frac{1}{2}}\right) \approx \frac{3}{8} u\left(t_{n+1}, x_{0}\right)+\frac{3}{4} u\left(t_{n+1}, x_{1}\right)-\frac{1}{8} u\left(t_{n+1}, x_{2}\right),
$$

where

$$
u\left(t_{n+1}, x_{\frac{1}{2}}\right)-\left(\frac{3}{8} u\left(t_{n+1}, x_{0}\right)+\frac{3}{4} u\left(t_{n+1}, x_{1}\right)-\frac{1}{8} u\left(t_{n+1}, x_{2}\right)\right)=R_{1}^{(2)}(\xi),
$$

and $R_{1}^{(2)}(\xi)=\frac{1}{16} u^{\prime \prime \prime}\left(t_{n+1}, c_{2}\right) h^{3}, c_{2} \in\left(0, x_{2}\right)$.

We then have

$$
\frac{1}{\Gamma(-\alpha)} \int_{0}^{x_{1}}\left(x_{2 j+1}-\xi\right)^{-1-\alpha} u\left(t_{n+1}, \xi\right) d \xi=\sum_{i=0}^{2} \hat{B}_{i} u\left(t_{n+1}, x_{i}\right)+R_{1},
$$

where

$$
\begin{aligned}
& \hat{B}_{0}=\int_{0}^{x_{1}}\left(x_{1}-\xi\right)^{\alpha-1} \frac{\left(\xi-x_{\frac{1}{2}}\right)\left(\xi-x_{1}\right)}{\left(x_{0}-x_{\frac{1}{2}}\right)\left(x_{0}-x_{1}\right)} d \xi+\frac{3}{8} \int_{0}^{x_{1}}\left(x_{1}-\xi\right)^{\alpha-1} \frac{\left(\xi-x_{0}\right)\left(\xi-x_{1}\right)}{\left(x_{\frac{1}{2}}-x_{0}\right)\left(x_{\frac{1}{2}}-x_{1}\right)} d \xi, \\
& \hat{B}_{1}=\frac{3}{4} \int_{0}^{x_{1}}\left(x_{1}-\xi\right)^{\alpha-1} \frac{\left(\xi-x_{0}\right)\left(\xi-x_{1}\right)}{\left(x_{\frac{1}{2}}-x_{0}\right)\left(x_{\frac{1}{2}}-x_{1}\right)} d \xi+\int_{0}^{x_{1}}\left(x_{1}-\xi\right)^{\alpha-1} \frac{\left(\xi-x_{0}\right)\left(\xi-x_{1}\right)}{\left(x_{1}-x_{0}\right)\left(x_{1}-x_{\frac{1}{2}}\right)} d \xi, \\
& \hat{B}_{2}=-\frac{1}{8} \int_{0}^{x_{1}}\left(x_{1}-\xi\right)^{\alpha-1} \frac{\left(\xi-x_{0}\right)\left(\xi-x_{1}\right)}{\left(x_{\frac{1}{2}}-x_{0}\right)\left(x_{\frac{1}{2}}-x_{1}\right)} d \xi,
\end{aligned}
$$

and

$$
R_{1}=\int_{0}^{x_{1}}\left(x_{2 j+1}-\xi\right)^{-1-\alpha} R_{1}^{(1)}(\xi) d \xi+\int_{0}^{x_{1}}\left(x_{2 j+1}-\xi\right)^{-1-\alpha} R_{1}^{(2)}(\xi) d \xi
$$

It is easy to show that

$$
\begin{aligned}
\left|R_{1}\right| & \leq \int_{0}^{x_{1}}\left(x_{2 j+1}-\xi\right)^{-1-\alpha}\left|R_{1}^{(1)}(\xi)\right| d \xi+\int_{0}^{x_{1}}\left(x_{2 j+1}-\xi\right)^{-1-\alpha}\left|R_{1}^{(2)}(\xi)\right| d \xi \\
& \leq \int_{0}^{x_{1}}\left(x_{2 j+1}-\xi\right)^{-1-\alpha} C \Delta x^{3} d \xi \leq C \Delta x^{3} \Delta x^{-\alpha}=C \Delta x^{3-\alpha} .
\end{aligned}
$$

Hence, we have

$$
\int_{0}^{x_{1}}\left(x_{2 j+1}-\xi\right)^{-1-\alpha} u\left(t_{n+1}, \xi\right) d \xi-\sum_{i=0}^{2} \hat{B}_{i} u_{i}^{n+1}=O\left(\Delta x^{3-\alpha}\right) .
$$

Similarly, we have, for some suitable weights $\tilde{B}_{i}, i=0,1,2$,

$$
\int_{x_{M-1}}^{x_{M}}\left(\xi-x_{2 j+1}\right)^{-1-\alpha} u\left(t_{n+1}, \xi\right) d \xi-\sum_{i=M-2}^{M} \tilde{B}_{i} U_{i}^{n+1}=O\left(\Delta x^{3-\alpha}\right) .
$$


Based on the analysis above, we will define $Q_{2 j}^{n+1}$ in (28) by

$$
Q_{2 j}^{n+1}=\frac{1}{\Gamma(-\alpha)} \sum_{i=0}^{2} \hat{B}_{i} U_{i}^{n+1}+\frac{1}{\Gamma(-\alpha)} \sum_{i=M-2}^{M} \tilde{B}_{i} U_{i}^{n+1} .
$$

It is easy to see that

$$
S_{2 j}^{n+1}-\frac{1}{\Gamma(-\alpha)}\left(\sum_{i=0}^{2} \hat{B}_{i} u_{i}^{n+1}+\sum_{i=M-2}^{M} \tilde{B}_{i} u_{i}^{n+1}\right)=O\left(\Delta x^{3-\alpha}\right) .
$$

Theorem 2.2. Let $1<\alpha<2$ and let $u\left(t_{n+1}, x_{l}\right)$ and $U_{l}^{n+1}, l=1,2, \ldots, M-1$ be the solutions of (25)(26) and (28)-(29), respectively. Assume that $u(t, \cdot) \in C^{3}[0,1]$. Further assume that $u(t, x)$ satisfies the Lipschitz conditions with the second variable, that is, with some $\beta>0$,

$$
\begin{aligned}
& \left|{ }_{0}^{R} D_{x}^{\alpha} u(t, x)-{ }_{0}^{R} D_{x}^{\alpha} u(t, y)\right| \leq C_{\alpha}|x-y|^{\beta}, \\
& \left|{ }_{x}^{R} D_{1}^{\alpha} u(t, x)-{ }_{x}^{R} D_{1}^{\alpha} u(t, y)\right| \leq C_{\alpha}|x-y|^{\beta} .
\end{aligned}
$$

Then

$$
\max _{1 \leq l \leq M-1}\left|u\left(t_{n+1}, x_{l}\right)-U_{l}^{n+1}\right| \leq C\left(\Delta t+\Delta x^{\min (\beta, 3-\alpha)}\right) .
$$

To prove this theorem, we need the following lemma.

Lemma 2.3. Let $1<\alpha<2$. The coefficients $w_{l, 2 j}$ in (13) satisfy

$$
\begin{aligned}
& w_{1,2 j}<0, \\
& w_{l, 2 j}>0, \quad l \neq 1, \quad l=0,2,3, \ldots, 2 j, \\
& \Gamma(3-\alpha) \sum_{k=0}^{2 j} w_{l, 2 j}<0 .
\end{aligned}
$$

Proof: It is easy to show that $w_{0,2 j}>0$ and $w_{1,2 j}<0$. We now prove that $w_{k, 2 j}>0, k=2,3, \ldots, 2 j$. We first show that

$$
w_{2 l-1,2 j}>0, \quad l=2,3, \ldots, j .
$$

Note that

$$
\Gamma(3-\alpha) w_{2 l-1,2 j}=2\left((2 l-2)^{-\alpha+2}-(2 l)^{-\alpha+2}\right)+2(-\alpha+2)\left((2 l-2)^{-\alpha+1}+(2 l)^{-\alpha+1}\right) .
$$

Let $m=2 l$. It is sufficient to show that, with $m=4,6, \ldots$,

$$
I(m)=(m-2)^{-\alpha+2}-m^{-\alpha+2}+(-\alpha+2)(m-2)^{-\alpha+1}+(-\alpha+2) m^{-\alpha+1}>0 .
$$

In fact, we have, by using a binomial expansion,

$$
\begin{aligned}
I(m)= & m^{-\alpha+2}\left(-1+(-\alpha+2) \frac{1}{m}+\left(1-\frac{2}{m}\right)^{-\alpha+2}+(-\alpha+2)\left(1-\frac{2}{m}\right)^{-\alpha+1} \frac{1}{m}\right) \\
& =m^{-\alpha+2}\left(\frac{(-\alpha+2)(-\alpha+1)(-\alpha)}{m^{3}}\left(-\frac{2^{3}}{3 !}+\frac{2^{2}}{2 !}\right)\right. \\
& +\frac{(-\alpha+2)(-\alpha+1)(-\alpha)(-\alpha-1)}{m^{4}}\left(\frac{2^{4}}{4 !}-\frac{2^{3}}{3 !}\right) \\
& \left.+\frac{(-\alpha+2)(-\alpha+1)(-\alpha)(-\alpha-1)}{m^{5}}\left(\frac{-2^{5}}{5 !}+\frac{2^{4}}{4 !}\right)+\ldots\right) .
\end{aligned}
$$


Note that the sequence $a_{n}=\frac{2^{n}}{n !}$ is decreasing. Hence we see that, with $1<\alpha<2$,

$$
I(m)>0 .
$$

We next prove that

$$
w_{2 l, 2 j}>0, l=1,2, \ldots, j-1
$$

Note that, with $l=2,3, \ldots, j-1$,

$$
\begin{aligned}
\Gamma(3-\alpha) w_{2 l, 2 j} & =-3(-\alpha+2)(2 l)^{-\alpha+1}+\left((2 l+2)^{-\alpha+2}-(2 l-2)^{-\alpha+2}\right) \\
& -\frac{1}{2}(-\alpha+2)\left((2 l+2)^{-\alpha+1}+(2 l-2)^{-\alpha+1}\right) .
\end{aligned}
$$

Let $m=2 l$. It is sufficient to show that, with $m=4,6, \ldots$,

$$
\begin{aligned}
I(m) & =-6(-\alpha+2) m^{-\alpha+1}+(-2)(m-2)^{-\alpha+2} \\
& +(-1)(-\alpha+2)(m-2)^{-\alpha+1}+2(m+2)^{-\alpha+2}+(-1)(-\alpha+2)(m+2)^{-\alpha+1}>0 .
\end{aligned}
$$

In fact, we have, by using a binomial expansion,

$$
\begin{aligned}
& I(m)=m^{-\alpha+2}\left(+(-2)\left(1-\frac{2}{m}\right)^{-\alpha+2}+(-1)(-\alpha+2)\left(1-\frac{2}{m}\right)^{-\alpha+1} \frac{1}{m}\right. \\
& \left.+2\left(1+\frac{2}{m}\right)^{-\alpha+2}+(-1)(-\alpha+2)\left(1+\frac{2}{m}\right)^{-\alpha+1} \frac{1}{m}\right) \\
& =m^{-\alpha+2}\left(\frac{2(-\alpha+2)(-\alpha+1)(-\alpha)(-\alpha-1)(-\alpha-2)}{m^{3}}\left(-\frac{2^{3} \cdot 2}{3 !}+\frac{2^{2}}{2 !}\right)\right. \\
& +\frac{2(-\alpha+2)(-\alpha+1)(-\alpha)(-\alpha-1)(-\alpha-2)}{m^{5}}\left(\frac{2^{5} \cdot 2}{5 !}-\frac{2^{4}}{4 !}\right) \\
& \left.+\frac{2(-\alpha+2)(-\alpha+1)(-\alpha)(-\alpha-1)(-\alpha-2)}{m^{7}}\left(\frac{2^{7} \cdot 2}{7 !}-\frac{2^{6}}{6 !}\right)+\ldots\right) \\
& =\frac{1}{m^{1+\alpha}}\left(\frac{2(-\alpha+2)(-\alpha+1)(-\alpha)}{m^{0}}\left(\frac{2^{3} \cdot 2}{3 !}-\frac{2^{2}}{2 !}\right)\right. \\
& +\frac{2(-\alpha+2)(-\alpha+1)(-\alpha)(-\alpha-1)(-\alpha-2)}{m^{2}}\left(\frac{2^{5} \cdot 2}{5 !}-\frac{2^{4}}{4 !}\right) \\
& +\frac{2(-\alpha+2)(-\alpha+1)(-\alpha)(-\alpha-1)(-\alpha-2)(-\alpha-3)(-\alpha-4)}{m^{4}} \\
& \left(\frac{2^{7} \cdot 2}{7 !}-\frac{2^{6}}{6 !}+\ldots\right)
\end{aligned}
$$

Note that

$$
\frac{2^{n} \cdot 2}{n !}-\frac{2^{n-1}}{(n-1) !}=\frac{2^{n-1}}{(n-1) !}\left(\frac{2 \cdot 2}{n}-1\right) \leq 0, n \geq 4 .
$$

Hence we obtain

$$
\begin{aligned}
I(m) \geq & \frac{2^{1+\alpha}}{m^{1+\alpha}} \frac{1}{2^{1+\alpha}}\left(\frac{2(-\alpha+2)(-\alpha+1)(-\alpha)}{m^{0}}\left(\frac{2^{3} \cdot 2}{3 !}-\frac{2^{2}}{2 !}\right)\right. \\
& +\frac{2(-\alpha+2)(-\alpha+1)(-\alpha)(-\alpha-1)(-\alpha-2)}{2^{2}}\left(\frac{2^{5} \cdot 2}{5 !}-\frac{2^{4}}{4 !}\right) \\
& +\frac{2(-\alpha+2)(-\alpha+1)(-\alpha)(-\alpha-1)(-\alpha-2)(-\alpha-3)(-\alpha-4)}{2^{4}}\left(\frac{2^{7} \cdot 2}{7 !}-\frac{2^{6}}{6 !}+\ldots\right) \\
= & \frac{2^{1+\alpha}}{m^{1+\alpha}} I(2), \quad m=2,3,4, \ldots
\end{aligned}
$$


It is easy to show that, with $1<\alpha<2$,

$$
I(2)=2^{-\alpha+2}\left(3 \alpha-6+2^{-\alpha}(6+\alpha)\right)>0 .
$$

Thus we obtain

$$
I(m)>0, \quad m=2,3,4, \ldots
$$

Similarly we can show that $w_{2 j, 2 j}>0$.

Finally we shall prove $\Gamma(3-\alpha) \sum_{l=0}^{2 j} w_{l, 2 j}<0$. We have

$$
\Gamma(3-\alpha) \sum_{l=0}^{2 j} w_{l, 2 j}=\frac{-3(-\alpha+2)}{2}(2 j)^{-\alpha+1}-(2 j)^{-\alpha+2}+\frac{\alpha-2}{2}(2 j+2)^{-\alpha+1}+(2 j+2)^{-\alpha+2} .
$$

Let $m=2 j+2$, it is sufficient to show that, with $m=4,6,8, \ldots$,

$$
I(m)=-3(-\alpha+2)(m-2)^{-\alpha+1}-2(m-2)^{-\alpha+2}+(\alpha-2) m^{-\alpha+1}+2 \cdot m^{-\alpha+2}<0 .
$$

In fact, by using a binomial expansion, we have

$$
\begin{aligned}
I(m)= & m^{-\alpha+2}\left((3 \alpha-6)\left(1-\frac{2}{m}\right)^{-\alpha+1} \frac{1}{m}-2\left(1-\frac{2}{m}\right)^{-\alpha+2}+(\alpha-2) \frac{1}{m}+2\right) \\
= & \frac{(-\alpha+2)(-\alpha+1)}{m^{2}}\left((-3) \frac{(-2)}{1 !}+(-2) \frac{(-2)^{2}}{2 !}\right) \\
& +\frac{(-\alpha+2)(-\alpha+1)(-\alpha)}{m^{3}}\left((-3) \frac{(-2)^{2}}{2 !}+(-2) \frac{(-2)^{3}}{3 !}\right. \\
& +\frac{(-\alpha+2)(-\alpha+1)(-\alpha)(-\alpha-1)}{m^{4}}\left((-3) \frac{(-2)^{3}}{3 !}+(-2) \frac{(-2)^{4}}{4 !}\right)+\ldots
\end{aligned}
$$

Note that

$$
\begin{aligned}
& (-3) \frac{(-2)^{n}}{n !}+(-2) \frac{\left.(-2)^{(} n+1\right)}{(n+1) !}=\frac{(-2)^{n}}{n !}\left((-3)+(-2) \frac{-2}{n+1}\right) \\
& =\frac{(-2)^{n}}{n !}\left((-3)+\frac{4}{n+1}\right)=\frac{-3 n+1}{(n+1) !}(-2)^{n}
\end{aligned}
$$

which implies that

$$
I(m)<0, m=4,6,8, \ldots
$$

Together these estimates complete the proof of Lemma 2.3.

Proof of Theorem 2.2: $\quad$ Let $e_{l}^{n+1}=u\left(t_{n+1}, x_{l}\right)-U_{l}^{n+1}, l=1,2, \ldots, M-1$. Subtracting (25) - (26) from (28)-(29), We obtain the following error equation, for $l=2 j, j=1,2 \ldots, m-1$, with $R=$ $O\left(\Delta t+\Delta x^{\min (3-\alpha, \beta)}\right)$,

$$
\Delta t^{-1}\left(e_{2 j}^{n+1}-e_{2 j}^{n}\right)-\Delta x^{-\alpha}\left(\sum_{k=0}^{2 j} w_{k, 2 j+1} e_{2 j+1-k}^{n+1}+\sum_{k=0}^{M-(2 j-1)-1} w_{k, M-(2 j-1)} e_{2 j-1+k}^{n+1}\right)+R
$$

and, for $l=2 j+1, j=0,1,2 \ldots, m-1$,

$$
\Delta t^{-1}\left(e_{2 j+1}^{n+1}-e_{2 j+1}^{n}\right)-\Delta x^{-\alpha}\left(\sum_{k=0}^{2 j+2} w_{k, M-(2 j+2)} e_{2 j+2+k}^{n+1}+\sum_{k=0}^{M-2 j} w_{k, M-2 j} e_{2 j+k}^{n+1}\right)+R .
$$


With $\lambda=\Delta t / \Delta x^{\alpha}$, we have, for $l=2 j, j=1,2 \ldots, m-1$,

$$
\begin{aligned}
& \left(1-\lambda w_{1,2 j+1}-\lambda w_{1, M-(2 j-1)}\right) e_{2 j}^{n+1} \\
& -\lambda\left(w_{0,2 j+1} e_{2 j+1}^{n+1}+\quad+w_{2,2 j+1} e_{2 j-1}^{n+1}+\cdots+w_{2 j, 2 j+1} e_{1}^{n+1}\right) \\
& -\lambda\left(w_{0, M-(2 j-1)} e_{2 j-1}^{n+1}+\quad+w_{2, M-(2 j-1)} e_{2 j+1}^{n+1}+\cdots+w_{M-(2 j-1)-1, M-(2 j-1)} e_{M-1}^{n+1}\right) \\
= & e_{2 j}^{n}+\Delta t R,
\end{aligned}
$$

and, for $l=2 j+1, j=0,1,2 \ldots, m-1$,

$$
\begin{aligned}
& \left(1-\lambda w_{1,2 j+2}-\lambda w_{1, M-2 j}\right) e_{2 j+1}^{n+1} \\
& -\lambda\left(w_{0,2 j+2} e_{2 j+2}^{n+1}+\quad+w_{2,2 j+2} e_{2 j}^{n+1}+\cdots+w_{2 j+2,2 j+2} e_{0}^{n+1}\right) \\
& -\lambda\left(w_{0, M-2 j} e_{2 j}^{n+1}+\quad+w_{2, M-2 j} e_{2 j+2}^{n+1}+\cdots+w_{M-2 j, M-2 j} e_{M}^{n+1}\right) \\
= & e_{2 j+1}^{n}+\Delta t R .
\end{aligned}
$$

Assume that $\left|e^{1}\right|_{\infty}=\sup _{l}\left|e_{l}^{1}\right|=\left|e_{2 k}\right|$ for some $k$, we obtain, by Lemma 2.3 , with $R=\left(\Delta t+\Delta x^{\min (3-\alpha, \beta)}\right)$,

$$
\begin{aligned}
& \left|e^{1}\right|_{\infty}=\sup _{l}\left|e_{l}^{1}\right|=\left|e_{2 k}^{1}\right| \leq\left|e_{2 k}^{1}\right|\left(1-\lambda\left(w_{0,2 k+1}+w_{1,2 k+1}+\ldots w_{2 k, 2 k+1}\right)\right. \\
& \left.\quad-\lambda\left(w_{0, M-(2 k-1)}+w_{1, M-(2 k-1)}+\cdots+w_{M-(2 k-1)-1, M-(2 k-1)}\right)\right) \\
& \leq\left|e_{2 k}^{1}\right|-\lambda w_{0,2 k+1}\left|e_{2 k+1}^{1}\right|-\lambda w_{1,2 k+1}\left|e_{2 k}^{1}\right|-\cdots-\lambda w_{2 k, 2 k+1}\left|e_{1}^{1}\right| \\
& \left.-\lambda w_{0, M-(2 k-1)}\left|e_{2 k-1}^{1}\right|-\lambda w_{1, M-(2 k-1)}\left|e_{2 k}^{1}\right|-\cdots-\lambda w_{M-(2 k-1)-1, M-(2 k-1)}\right)\left|e_{M-1}^{1}\right| \\
& \leq\left|e_{2 k}^{1}\right|-\lambda w_{0,2 k+1}\left|e_{2 k+1}^{1}\right|-\lambda w_{1,2 k+1}\left|e_{2 k}^{1}\right|-\cdots-\lambda w_{2 k, 2 k+1}\left|e_{1}^{1}\right| \\
& \quad-\lambda w_{0, M-(2 k-1)}\left|e_{2 k-1}^{1}\right|-\lambda w_{1, M-(2 k-1)}\left|e_{2 k}^{1}\right|-\cdots-\lambda w_{M-(2 k-1)-1, M-(2 k-1)}\left|e_{M-1}^{1}\right| \\
& \leq\left|e_{2 k}^{0}\right|+\Delta t R .
\end{aligned}
$$

Assume that $\left|e^{1}\right|_{\infty}=\sup _{l}\left|e_{l}^{1}\right|=\left|e_{2 k+1}\right|$ for some $k$, we obtain, by Lemma 2.3, with $R=(\Delta t+$ $\left.\Delta x^{\min (3-\alpha, \beta)}\right)$,

$$
\begin{aligned}
\left|e^{1}\right|_{\infty}=\sup _{l}\left|e_{l}^{1}\right|= & \left|e_{2 k+1}^{1}\right| \leq\left|e_{2 k+1}^{1}\right|\left(1-\lambda\left(w_{0,2 k+2}+w_{1,2 k+2}+\ldots w_{2 k+2,2 k+2}\right)\right. \\
& \left.\quad-\lambda\left(w_{0, M-2 k}+w_{1, M-2 k}+\cdots+w_{M-2 k, M-2 k}\right)\right) \\
= & \left|e_{2 k+1}^{1}\right|-\lambda w_{0,2 k+2}\left|e_{2 k+2}^{1}\right|-\lambda w_{1,2 k+2}\left|e_{2 k+1}^{1}\right|-\cdots-\lambda w_{2 k+2,2 k+2}\left|e_{0}^{1}\right| \\
- & \left.\lambda w_{0, M-2 k}\left|e_{2 k}^{1}\right|-\lambda w_{1, M-2 k}\left|e_{2 k+1}^{1}\right|+\cdots-\lambda w_{M-2 k, M-2 k}\right)\left|e_{M}^{1}\right| \\
\leq & \left|e_{2 k+1}^{1}\right|-\lambda w_{0,2 k+2}\left|e_{2 k+1}^{1}\right|-\lambda w_{1,2 k+2}\left|e_{2 k+1}^{1}\right|-\cdots-\lambda w_{2 k+2,2 k+2}\left|e_{0}^{1}\right| \\
& \quad-\lambda w_{0, M-2 k}\left|e_{2 k}^{1}\right|-\lambda w_{1, M-2 k}\left|e_{2 k+1}^{1}\right|-\cdots-\lambda w_{M-2 k, M-2 k}\left|e_{M}^{1}\right| \\
\leq & \left|e_{2 k+1}^{0}\right|+\Delta t R .
\end{aligned}
$$

Hence we obtain

$$
\sup _{l}\left|e_{l}^{1}\right| \leq \sup _{l}\left|e_{l}^{0}\right|+\Delta t R
$$

Further, for simplicity, we assume that $e_{l}^{0}=0$. Then we have

$$
\left|e^{1}\right|_{\infty} \leq \Delta t R
$$

Similarly, we can show that

$$
\left|e^{2}\right|_{\infty} \leq\left|e^{1}\right|_{\infty}+\Delta t R \leq t_{2} R
$$


and in general, with $0 \leq t_{n} \leq T$,

$$
\left|e^{n}\right|_{\infty} \leq t_{n} R \leq C\left(\Delta t+\Delta x^{\min (3-\alpha, \beta)}\right) .
$$

The proof of Theorem 2.2 is now complete.

\section{Numerical simulations}

In this section, we will give some numerical examples. Let us consider the following space-fractional partial differential equation with nonhomogeneous Dirichlet boundary conditions, with $1<\alpha<2$,

$$
\begin{aligned}
& \frac{\partial u(t, x)}{\partial t}-{ }_{0}^{R} D_{x}^{\alpha} u(t, x)=f(t, x), \quad 0<x<1, t>0, \\
& u(t, 0)=\varphi_{1}(t), \quad u(t, 1)=\varphi_{2}(t), \\
& u(0, x)=u_{0}(x),
\end{aligned}
$$

where $\varphi_{1}(t), \varphi_{2}(t)$ are some suitable functions of $t$ and $u_{0}(x)$ is the initial condition.

Let us recall the numerical method introduced in the previous section. Let $m$ be a positive integer and let $0=x_{0}<x_{1}<x_{2}<\cdots<x_{2 m}=1$ be a space partition of $[0,1]$ and $\Delta x$ the space stepsize. Let $0=t_{0}<t_{1}<t_{2}<\cdots<x_{N}=1$ be a time partition of $[0,1]$ and $\Delta t$ the time stepsize.

At $x=x_{l}, t=t_{n}$, we have, with $l=1,2, \ldots, 2 m-1$, and $n=1,2, \ldots, N$,

$$
\begin{aligned}
& \left.\frac{\partial u(t, x)}{\partial t}\right|_{x=x_{l}, t=t_{n}}-\left.{ }_{0}^{R} D_{x}^{\alpha} u(t, x)\right|_{x=x_{l}, t=t_{n}}=\left.f(t, x)\right|_{x=x_{l}, t=t_{n}}, \\
& u\left(t_{n}, 0\right)=\varphi_{1}\left(t_{n}\right), \quad u\left(t_{n}, 1\right)=\varphi_{2}\left(t_{n}\right), \\
& u\left(0, x_{l}\right)=u_{0}\left(x_{l}\right),
\end{aligned}
$$

To get a stable finite difference scheme for this time-dependent problem, we need to consider the following shifted equation, that is,

$$
\begin{aligned}
& \left.\frac{\partial u(t, x)}{\partial t}\right|_{x=x_{l}, t=t_{n}}-\left.{ }_{0}^{R} D_{x}^{\alpha} u(t, x)\right|_{x=x_{l+1}, t=t_{n}}=\left.f(t, x)\right|_{x=x_{l}, t=t_{n}}+\rho_{l}\left(t_{n}\right), \\
& u\left(t_{n}, 0\right)=\varphi_{1}\left(t_{n}\right), \quad u\left(t_{n}, 1\right)=\varphi_{2}\left(t_{n}\right), \\
& u\left(0, x_{l}\right)=u_{0}\left(x_{l}\right),
\end{aligned}
$$

where

Note that,

$$
\rho_{l}\left(t_{n}\right)=-\left(\left.{ }_{0}^{R} D_{x}^{\alpha} u(t, x)\right|_{x=x_{l+1}, t=t_{n}}-\left.{ }_{0}^{R} D_{x}^{\alpha} u(t, x)\right|_{x=x_{l}, t=t_{n}}\right) .
$$

$$
\left.\frac{\partial u(t, x)}{\partial t}\right|_{x=x_{l}, t=t_{n}}=\frac{u\left(t_{n}, x_{l}\right)-u\left(t_{n-1}, x_{l}\right)}{\Delta t}+O(\Delta t),
$$

and, with $l=2 j, j=1,2, \ldots, m-1$,

$$
\begin{aligned}
\left.{ }_{0}^{R} D_{x}^{\alpha} u(t, x)\right|_{x=x_{l+1}, t=t_{n}} & =\frac{1}{\Gamma(-\alpha)} \int_{x_{0}}^{x_{2 j+1}}\left(x_{2 j+1}-\xi\right)^{-1-\alpha} u\left(t_{n}, \xi\right) d \xi \\
& =\frac{1}{\Gamma(-\alpha)} \int_{0}^{x_{1}}\left(x_{2 j+1}-\xi\right)^{-1-\alpha} u\left(t_{n}, \xi\right) d \xi \\
& +\Delta x^{-\alpha} \sum_{k=0}^{2 j} w_{k, 2 j+1} u\left(t_{n}, x_{2 j+1-k}\right)+O\left(\Delta x^{3-\alpha}\right)
\end{aligned}
$$


and, with $l=2 j+1, j=0,1,2, \ldots, m-1$,

$$
\begin{aligned}
\left.{ }_{0}^{R} D_{x}^{\alpha} u(t, x)\right|_{x=x_{l+1}, t=t_{n}} & =\frac{1}{\Gamma(-\alpha)} \int_{0}^{x_{2 j+2}}\left(x_{2 j+2}-\xi\right)^{-1-\alpha} u\left(t_{n}, \xi\right) d \xi \\
& =\Delta x^{-\alpha} \sum_{k=0}^{2 j+2} w_{k, 2 j+2} u\left(t_{n}, x_{2 j+2-k}\right)+O\left(\Delta x^{3-\alpha}\right),
\end{aligned}
$$

where $w_{k, 2 j+1}, w_{k, 2 j+2}$ are defined as in (13) and (14).

Denote $U_{j}^{n} \approx u\left(t_{n}, x_{j}\right)$. We define the following backward Euler method for solving (36)-(38),

$$
\begin{aligned}
& \frac{U_{2 j}^{n}-U_{2 j}^{n-1}}{\Delta t}- \Delta x^{-\alpha} \sum_{k=0}^{2 j} w_{k, 2 j+1} U_{2 j+1-k}^{n}=f\left(x_{2 j}, t_{n}\right)+\rho_{2 j}^{n} \\
&+\frac{1}{\Gamma(-\alpha)} \int_{0}^{x_{1}}\left(x_{2 j+1}-\xi\right)^{-1-\alpha} u\left(\xi, t_{n}\right) d \xi, j=1,2, \ldots, m-1, \\
& \frac{U_{2 j+1}^{n}-U_{2 j+1}^{n-1}}{\Delta t}-\Delta x^{-\alpha} \sum_{k=0}^{2 j+2} w_{k, 2 j+2} U_{2 j+2-k}^{n}=f\left(x_{2 j+1}, t_{n}\right)+\rho_{2 j+1}^{n} \\
&+\frac{1}{\Gamma(-\alpha)} \int_{0}^{x_{1}}\left(x_{2 j+1}-\xi\right)^{-1-\alpha} u\left(\xi, t_{n}\right) d \xi, j=0,1,2, \ldots, m-1,
\end{aligned}
$$

or, with $\lambda=\frac{\Delta t}{\Delta x^{\alpha}}$,

$$
\begin{aligned}
& U_{2 j}^{n}-\lambda \sum_{k=0}^{2 j} w_{k, 2 j+1} U_{2 j+1-k}^{n}=U_{2 j}^{n-1}+\Delta t f\left(x_{2 j}, t_{n}\right)+\Delta t \rho_{2 j}^{n} \\
& \quad+\Delta t \frac{1}{\Gamma(-\alpha)} \int_{0}^{x_{1}}\left(x_{2 j+1}-\xi\right)^{-1-\alpha} u\left(\xi, t_{n}\right) d \xi, \quad j=1,2, \ldots, m-1, \\
& U_{2 j+1}^{n}-\lambda \sum_{k=0}^{2 j+2} w_{k, 2 j+2} U_{2 j+2-k}^{n}=U_{2 j+1}^{n-1}+\Delta t f\left(x_{2 j+1}, t_{n}\right)+\Delta t \rho_{2 j+1}^{n}, \quad j=0,1,2, \ldots, M-1 .
\end{aligned}
$$

The numerical methods (45) - (46) can be written into the following matrix form

$$
A U^{n}=U^{n}+\Delta t F^{n}+\Delta t \rho^{n}+\Delta t I^{n}+B_{l}^{n}+B_{r}^{n},
$$

where

$$
U^{n}=\left[\begin{array}{c}
U_{1}^{n} \\
U_{2}^{n} \\
U_{3}^{n} \\
\vdots \\
U_{2 m-1}^{n}
\end{array}\right], \quad F^{n}=\left[\begin{array}{c}
f\left(x_{1}, t_{n}\right) \\
f\left(x_{2}, t_{n}\right) \\
f\left(x_{3}, t_{n}\right) \\
\vdots \\
f\left(x_{2 m-1}, t_{n}\right)
\end{array}\right], \quad \rho^{n}=\left[\begin{array}{c}
-\left({ }_{0}^{R} D_{x}^{\alpha} u\left(x_{2}, t_{n}\right)-{ }_{0}^{R} D_{x}^{\alpha} u\left(x_{1}, t_{n}\right)\right) \\
-\left({ }_{0}^{R} D_{x}^{\alpha} u\left(x_{3}, t_{n}\right)-{ }_{0}^{R} D_{x}^{\alpha} u\left(x_{2}, t_{n}\right)\right) \\
-\left({ }_{0}^{R} D_{x}^{\alpha} u\left(x_{4}, t_{n}\right)-{ }_{0}^{R} D_{x}^{\alpha} u\left(x_{3}, t_{n}\right)\right) \\
\vdots \\
-\left({ }_{0}^{R} D_{x}^{\alpha} u\left(x_{2 m}, t_{n}\right)-{ }_{0}^{R} D_{x}^{\alpha} u\left(x_{2 m-1}, t_{n}\right)\right)
\end{array}\right],
$$

and

$$
I^{n}=\left[\begin{array}{c}
0 \\
\frac{1}{\Gamma(-\alpha)} \int_{0}^{x_{1}}\left(x_{3}-\xi\right)^{-1-\alpha} u\left(t_{n}, \xi\right) d \xi \\
0 \\
\vdots \\
\frac{1}{\Gamma(-\alpha)} \int_{0}^{x_{1}}\left(x_{2 m-1}-\xi\right)^{-1-\alpha} u\left(t_{n}, \xi\right) d \xi \\
0
\end{array}\right], B_{l}^{n}=\left[\begin{array}{c}
\lambda w_{2,2} u\left(t_{n}, x_{0}\right) \\
0 \\
\lambda w_{4,4} u\left(t_{n}, x_{0}\right) \\
0 \\
\vdots \\
0 \\
\lambda w_{2 m, 2 m} u\left(t_{n}, x_{0}\right)
\end{array}\right], B_{r}^{n}=\left[\begin{array}{c}
0 \\
0 \\
\vdots \\
0 \\
\lambda w_{0,2 m} u\left(t_{n}, x_{2 m}\right)
\end{array}\right]
$$


and

$$
A=\left[\begin{array}{cccccc}
1-\lambda w_{1,2} & -\lambda w_{0,2} & 0 & 0 & \cdots & 0 \\
-\lambda w_{2,3} & 1-\lambda w_{1,3} & -\lambda w_{0,3} & 0 & \cdots & 0 \\
\vdots & \vdots & \vdots & \vdots & \vdots & \vdots \\
-\lambda w_{2 m-2,2 m-1} & -\lambda w_{2 m-3,2 m-1} & \cdots & \cdots & 1-\lambda w_{1,2 m-1} & -\lambda w_{0,2 m-1} \\
-\lambda w_{2 m-1,2 m} & -\lambda w_{2 m-2,2 m} & \cdots & \cdots & -\lambda w_{2,2 m} & 1-\lambda w_{1,2 m}
\end{array}\right] .
$$

Here $B_{l}^{n}$ and $B_{r}^{n}$ are determined by the Dirichlet boundary conditions $u\left(t_{n}, x_{0}\right)$ and $u\left(t_{n}, x_{2 m}\right)$. We then use MATLAB to obtain all the approximate solutions $U^{n}, n=1,2, \ldots, N$.

Example 3.1. Consider [2]

$$
\begin{aligned}
& u_{t}(t, x)={ }_{0}^{R} D_{x}^{\alpha} u(t, x)+f(t, x), \quad 0<x<2, t>0 \\
& u(t, 0)=0, \quad u(t, 2)=0, \\
& u(0, x)=4 x^{2}(2-x)^{2}, \quad 0<x<1,
\end{aligned}
$$

where

$$
f(t, x)=-4 e^{-t} x^{2}(2-x)^{2}-4 e^{-t}\left(4 \frac{\Gamma(2+1)}{\Gamma(2-\alpha+1)} x^{2-\alpha}-4 \frac{\Gamma(3+1)}{\Gamma(3-\alpha+1)} x^{3-\alpha}+\frac{\Gamma(4+1)}{\Gamma(4-\alpha+1)} x^{4-\alpha},\right.
$$

The exact solution is $u(t, x)=4 e^{-t} x^{2}(2-x)^{2}$.

By Theorem 2.2, we have

$$
\left|e^{N}\right|_{\infty}=\left|U^{N}-u\left(t_{N}\right)\right|_{\infty} \leq C\left(\Delta t+\Delta x^{\gamma}\right), \text { with } \gamma=\min (3-\alpha, \beta),
$$

where $\left|e^{N}\right|_{\infty}$ denotes the $L^{\infty}$-norm of the error at time $t_{N}=1$. In our numerical example, we know the exact solution $u$, so we can exactly calculate $\rho^{n}$. In general, we may need to approximate $\rho^{n}$ by using the computed solutions $U^{n}$ with some higher order approximation numerical methods.

To observe the convergence order with respect to $\Delta x$, we choose $\Delta t=2^{-10}$ sufficiently small and the different space step sizes $h_{l}=\Delta x=2^{-l}, l=2,3,4,5,6$. Hence the error will be dominated by $\Delta x^{\gamma}$. Now let $\left|e_{l}^{N}\right|_{\infty}=\left|U^{N}-u\left(t_{N}\right)\right|_{\infty}$ denote the $L^{\infty}$-norm at $t_{N}=1$ obtained by using the space stepsize $h_{l}$. For the fixed space stepsize $h_{l}=2^{-l}, l=2,3,4,5,6$, we have

$$
\left|e_{l}^{N}\right|_{\infty} \approx C h_{l}^{\gamma},
$$

which implies that

$$
\frac{\left|e_{l}^{N}\right|_{\infty}}{\left|e_{l+1}^{N}\right|_{\infty}} \approx \frac{h_{l}^{\gamma}}{h_{l+1}^{\gamma}}=2^{\gamma}
$$

Hence the convergence order satisfies

$$
\gamma \approx \log _{2}\left(\frac{\left|e_{l}^{N}\right|_{\infty}}{\left|e_{l+1}^{N}\right|_{\infty}}\right)
$$

In Table 1, we obtain the experimentally determined orders of convergence (EOC) for the different $\alpha=$ $1.2,1.4,1.6,1.8$. We see that the convergence order is almost $3-\alpha$ which is consistent with our theoretical convergence order $\gamma=\min (3-\alpha, \beta)$. The order $3-\alpha$ term dominates the convergence order in this example. Here and below we will call our numerical method "the Shifted Diethelm method".

In [24], the shifted Grünwald difference operator

$$
A_{h, p}^{\alpha} u(x)=\Delta x^{-\alpha} \sum_{k=0}^{\infty} g_{k}^{(\alpha)} u(x-(k-p) \Delta x)
$$




\begin{tabular}{|c|c|c|c|c|c|}
\hline$\Delta t$ & $\Delta x$ & $\alpha=1.2$ & $\alpha=1.4$ & $\alpha=1.6$ & $\alpha=1.8$ \\
\hline $2^{-10}$ & $2^{-3}$ & & & & \\
\hline $2^{-10}$ & $2^{-4}$ & 1.5009 & 1.5203 & 1.4714 & 1.5419 \\
\hline $2^{-10}$ & $2^{-5}$ & 1.5813 & 1.4978 & 1.3432 & 1.3221 \\
\hline $2^{-10}$ & $2^{-6}$ & 1.7058 & 1.5597 & 1.3262 & 1.2168 \\
\hline $2^{-10}$ & $2^{-7}$ & 1.8136 & 1.6285 & 1.3504 & 1.1905 \\
\hline
\end{tabular}

Table 1: The experimentally determined orders of convergence (EOC) at $t=1$ in Example 3.1 by using the shifted Diethelm's method

approximates the Riemann-Liouville fractional derivative uniformly with first order accuracy, i.e.,

$$
A_{h, p}^{\alpha} u(x)={ }_{-\infty}^{R} D_{x}^{\alpha} u(x)+O(\Delta x),
$$

where $p$ is an positive integer and $g_{k}^{(\alpha)}=(-1)^{k}\left(\begin{array}{c}\alpha \\ k\end{array}\right)$. Considering a well defined function $u(x)$ on a bounded interval $[a, b]$ if $u(a)=0$ or $u(b)=0$, the function $u(x)$ can be zero extended for $x<a$ or $x>b$. And then the $\alpha$ order left and right Riemann-Liouville fractional derivatives of $u(x)$ at each point $x$ can be approximated by the shifted Grünwald difference operator $A_{h, p}^{\alpha} u(x)$. In [33], the authors introduced a weighted and shifted Grünwald difference operator which has second order accuracy to approximate the Riemann-Liouville fractional derivative. However the approximation of the left or right Riemann-Liouville fractional derivatives in [24], [33] by using the shifted Grünwald difference operator on finite interval $[a, b]$ requires that $u(a)=0$ or $u(b)=0$ respectively. In Table 2 , we obtain the experimentally determined orders of convergence (EOC) for the different $\alpha=1.2,1.4,1.6,1.8$ by using Grünwand difference method in [24]. We only observe the first order convergence.

\begin{tabular}{|c|c|c|c|c|c|}
\hline$\Delta t$ & $\Delta x$ & $\alpha=1.2$ & $\alpha=1.4$ & $\alpha=1.6$ & $\alpha=1.8$ \\
\hline $2^{-10}$ & $2^{-3}$ & & & & \\
\hline $2^{-10}$ & $2^{-4}$ & 0.8970 & 0.9660 & 1.1971 & 1.7665 \\
\hline $2^{-10}$ & $2^{-5}$ & 0.9304 & 0.9997 & 1.0878 & 1.4690 \\
\hline $2^{-10}$ & $2^{-6}$ & 0.9571 & 1.0004 & 1.0340 & 1.1946 \\
\hline $2^{-10}$ & $2^{-7}$ & 0.9792 & 1.0033 & 1.0166 & 1.0674 \\
\hline
\end{tabular}

Table 2: The experimentally determined orders of convergence (EOC) at $t=1$ in Example 3.1 by using the shifted Grünwald method

Example 3.2. We consider the same equation as in Example 3.1, but with the nonhomogenous Dirichlet boundary condition,

$$
\begin{aligned}
& u_{t}(t, x)={ }_{0}^{R} D_{x}^{\alpha} u(t, x)+f(t, x), \quad 0<x<2, t>0 \\
& u(t, 0)=5, \quad u(t, 2)=5, \\
& u(0, x)=4 x^{2}(2-x)^{2}+5, \quad 0<x<1,
\end{aligned}
$$

where

$f(t, x)=-4 e^{-t} x^{2}(2-x)^{2}-4 e^{-t}\left(4 \frac{\Gamma(2+1)}{\Gamma(2-\alpha+1)} x^{2-\alpha}-4 \frac{\Gamma(3+1)}{\Gamma(3-\alpha+1)} x^{3-\alpha}+\frac{\Gamma(4+1)}{\Gamma(4-\alpha+1)} x^{4-\alpha}+5 \frac{\Gamma(1)}{\Gamma(1-\alpha)} x^{-\alpha}\right)$.

The exact solution is $u(t, x)=4 e^{-t} x^{2}(2-x)^{2}+5$. 
We use the same notations as in Example 3.1. In Table 3, we obtain the experimentally determined orders of convergence (EOC) for the different $\alpha=1.2,1.4,1.6,1.8$. We see that the convergence order is less than $3-\alpha$. This is because of the nonhomogeneous boundary conditions.

Since the approximation of the Riemann-Liouville fractional derivative by using Grünwald difference operator on $[a, b]$ in Meerschaert and Tadjeran [24] requires that the function has the zero extension for $x<a$ and $x>b$. Hence we require that the function should have zero boundary conditions on the finite interval in order to get good approximation of the fractional derivative of such function by using the Grünald difference operator. In this example, since the Dirichlet boundary conditions are not homogenuous, we observe that in Table 4 the convergence order of the algorithm by using Grünwald difference method is rather low. However the shifted Diethelm's method works well for the nonhomogeneous Dirichlet boundary conditions and the convergence order is approximately equal to 1 in this example. This is another advantage by using the shifted Dietehlm's method compared with the Grünwald difference method in Meerschaert and Tadjeran [24].

\begin{tabular}{|c|c|c|c|c|c|}
\hline$\Delta t$ & $\Delta x$ & $\alpha=1.2$ & $\alpha=1.4$ & $\alpha=1.6$ & $\alpha=1.8$ \\
\hline $2^{-10}$ & $2^{-3}$ & & & & \\
\hline $2^{-10}$ & $2^{-4}$ & 1.4510 & 1.4687 & 1.5479 & 1.6511 \\
\hline $2^{-10}$ & $2^{-5}$ & 1.4388 & 1.2905 & 1.2426 & 1.2030 \\
\hline $2^{-10}$ & $2^{-6}$ & 1.3686 & 1.1039 & 0.9791 & 1.0037 \\
\hline $2^{-10}$ & $2^{-7}$ & 1.0667 & 0.8199 & 0.7011 & 0.7089 \\
\hline
\end{tabular}

Table 3: The experimentally determined orders of convergence (EOC) at $t=1$ in Example 3.2 by using the shifted Diethelm's method

\begin{tabular}{|c|c|c|c|c|c|}
\hline$\Delta t$ & $\Delta x$ & $\alpha=1.2$ & $\alpha=1.4$ & $\alpha=1.6$ & $\alpha=1.8$ \\
\hline $2^{-10}$ & $2^{-3}$ & & & & \\
\hline $2^{-10}$ & $2^{-4}$ & 0.7821 & 0.3548 & 0.6070 & 1.1859 \\
\hline $2^{-10}$ & $2^{-5}$ & 0.5424 & 0.2148 & 0.2738 & 0.5377 \\
\hline $2^{-10}$ & $2^{-6}$ & 0.4045 & 0.2604 & 0.2348 & 0.2664 \\
\hline $2^{-10}$ & $2^{-7}$ & 0.3801 & 0.3191 & 0.2580 & 0.1939 \\
\hline
\end{tabular}

Table 4: The experimentally determined orders of convergence (EOC) at $t=1$ in Example 3.2 by using the shifted Grünwald method

Example 3.3. Consider [2]

$$
\begin{aligned}
& u_{t}(t, x)={ }_{0}^{R} D_{x}^{\alpha} u(t, x)+f(t, x), \quad 0<x<1, t>0 \\
& u(t, 0)=0, \quad u(t, 1)=e^{-t}, \\
& u(0, x)=x^{\alpha_{1}}, \quad 0<x<1,
\end{aligned}
$$

where

$$
f(t, x)=-e^{-t} x^{\alpha_{1}}-e^{-t} \frac{\Gamma\left(\alpha_{1}+1\right)}{\Gamma\left(\alpha_{1}+1-\alpha\right)} x^{\alpha_{1}-\alpha} .
$$

The exact solution is $u(t, x)=e^{-t} x^{\alpha_{1}}$. In our numerical simulations, we first consider the nonsmooth solutions with $\alpha_{1}=\alpha$. we then consider the smooth solutions with $\alpha_{1}=3$. 
For the case $\alpha_{1}=\alpha$, we have

$$
{ }_{0}^{R} D_{x}^{\alpha}\left(x^{\alpha_{1}}\right)=D^{2}\left({ }_{0}^{R} D_{x}^{\alpha-2}\right)\left(x^{\alpha_{1}}\right)=D^{2} \frac{1}{\Gamma(2-\alpha)} \int_{0}^{x}(x-\tau)^{1-\alpha} \tau^{\alpha_{1}} d \tau=C D^{2}\left(x^{2}\right)=C,
$$

for some constant $C$, which implies that the following Lipschitz condition holds for any $\beta>0$,

$$
\left|{ }_{0}^{R} D_{x}^{\alpha} u(t, x)-{ }_{0}^{R} D_{y}^{\alpha} u(t, y)\right|=0 \leq C|x-y|^{\beta} .
$$

In Table 5, we obtain the experimentally determined orders of convergence (EOC) for the different $\alpha=1.2,1.4,1.6,1.8$. We see that the convergence order is less than $3-\alpha$. This is because the exact solution $u$ is not sufficiently smooth in this case.

\begin{tabular}{|c|c|c|c|c|c|}
\hline$\Delta t$ & $\Delta x$ & $\alpha=1.2, \alpha_{1}=1.2$ & $\alpha=1.4, \alpha_{1}=1.4$ & $\alpha=1.6, \alpha_{1}=1.6$ & $\alpha=1.8, \alpha_{1}=1.8$ \\
\hline $2^{-10}$ & $2^{-3}$ & & & & \\
\hline $2^{-10}$ & $2^{-4}$ & 1.2981 & 1.1479 & 1.0375 & 0.9583 \\
\hline $2^{-10}$ & $2^{-5}$ & 1.4639 & 1.3352 & 1.1884 & 1.0637 \\
\hline $2^{-10}$ & $2^{-6}$ & 1.4405 & 1.4178 & 1.2836 & 1.1379 \\
\hline $2^{-10}$ & $2^{-7}$ & 1.2192 & 1.4118 & 1.3292 & 1.1831 \\
\hline
\end{tabular}

Table 5: The experimentally determined orders of convergence (EOC) at $t=1$ in Example 3.3 for $\alpha_{1}=\alpha$

For the case $\alpha_{1}=3$, we obtain, in Table 6 , the experimentally determined orders of convergence (EOC) for the different $\alpha=1.2,1.4,1.6,1.8$. We see that the convergence order is almost $3-\alpha$.

\begin{tabular}{|c|c|c|c|c|c|}
\hline$\Delta t$ & $\Delta x$ & $\alpha=1.2, \alpha_{1}=3$ & $\alpha=1.4, \alpha_{1}=3$ & $\alpha=1.6, \alpha_{1}=3$ & $\alpha=1.8, \alpha_{1}=3$ \\
\hline $2^{-10}$ & $2^{-3}$ & & & & \\
\hline $2^{-10}$ & $2^{-4}$ & 1.3625 & 1.2416 & 1.1532 & 1.0745 \\
\hline $2^{-10}$ & $2^{-5}$ & 1.5740 & 1.3951 & 1.2398 & 1.1111 \\
\hline $2^{-10}$ & $2^{-6}$ & 1.7143 & 1.5008 & 1.3099 & 1.1440 \\
\hline $2^{-10}$ & $2^{-7}$ & 1.8557 & 1.5754 & 1.3585 & 1.1690 \\
\hline
\end{tabular}

Table 6: The experimentally determined orders of convergence (EOC) at $t=1$ in Example 3.3 for $\alpha_{1}=3$

Example 3.4. Consider the same equation as in Example 3.3, but with the nonhomogeneous boundary conditions.

$$
\begin{aligned}
& u_{t}(t, x)={ }_{0}^{R} D_{x}^{\alpha} u(t, x)+f(t, x), \quad 0<x<1, t>0 \\
& u(t, 0)=1, \quad u(t, 1)=e^{-t}+1, \\
& u(0, x)=x^{\alpha_{1}}+1, \quad 0<x<1,
\end{aligned}
$$

where

$$
f(t, x)=-e^{-t} x^{\alpha_{1}}-e^{-t} \frac{\Gamma\left(\alpha_{1}+1\right)}{\Gamma\left(\alpha_{1}+1-\alpha\right)} x^{\alpha_{1}-\alpha} .
$$

The exact solution is $u(t, x)=e^{-t} x^{\alpha_{1}}+1$. In our numerical simulations, we consider the smooth solution with $\alpha_{1}=3$. 
In Table 7, we obtain the experimentally determined orders of convergence (EOC) for the different $\alpha=1.2,1.4,1.6,1.8$. We see that the convergence order is almost $3-\alpha$ even under the nonhomogeneous boundary conditions.

\begin{tabular}{|c|c|c|c|c|c|}
\hline$\Delta t$ & $\Delta x$ & $\alpha=1.2, \alpha_{1}=3$ & $\alpha=1.4, \alpha_{1}=3$ & $\alpha=1.6, \alpha_{1}=3$ & $\alpha=1.8, \alpha_{1}=3$ \\
\hline $2^{-10}$ & $2^{-3}$ & & & & \\
\hline $2^{-10}$ & $2^{-4}$ & 1.3961 & 1.2732 & 1.1686 & 1.0791 \\
\hline $2^{-10}$ & $2^{-5}$ & 1.5847 & 1.4090 & 1.2514 & 1.1165 \\
\hline $2^{-10}$ & $2^{-6}$ & 1.7003 & 1.5015 & 1.3149 & 1.1474 \\
\hline $2^{-10}$ & $2^{-7}$ & 1.7823 & 1.5581 & 1.3562 & 1.1698 \\
\hline
\end{tabular}

Table 7: The experimentally determined orders of convergence (EOC) at $t=1$ in Example 3.4 for $\alpha_{1}=3$

Example 3.5. Consider the following two-sided space-fractional partial differential equation, [24]

$$
\begin{aligned}
& u_{t}(t, x)=c_{+}(t, x){ }_{0}^{R} D_{x}^{\alpha} u(t, x)+c_{-}(t, x){ }_{x}^{R} D_{1}^{\alpha} u(t, x)+f(t, x), \quad 0<x<2, t>0 \\
& u(t, 0)=u(t, 2)=0, \\
& u(0, x)=4 x^{2}(2-x)^{2}, \quad 0<x<2,
\end{aligned}
$$

where

$$
\begin{gathered}
c_{+}(t, x)=\Gamma(1.2) x^{1.8} \quad \text { and } \quad c_{-}(t, x)=\Gamma(1.2)(2-x)^{1.8} \\
f(t, x)=-32 e^{-t}\left(x^{2}+(2-x)^{2}-2.5\left(x^{3}+(2-x)^{3}\right)+\frac{25}{22}\left(x^{4}+(2-x)^{4}\right)\right) .
\end{gathered}
$$

The exact solution is $u(t, x)=4 e^{-t} x^{2}(2-x)^{2}$.

We use the same notations as in Example 3.1. In Table 8, we obtain the experimentally determined orders of convergence (EOC) for the different $\alpha=1.2,1.4,1.6,1.8$. We see that the convergence order is almost $3-\alpha$. The order $3-\alpha$ term dominates the convergence order in this example.

\begin{tabular}{|c|c|c|c|c|c|}
\hline$\Delta t$ & $\Delta x$ & $\alpha=1.2$ & $\alpha=1.4$ & $\alpha=1.6$ & $\alpha=1.8$ \\
\hline $2^{-10}$ & $2^{-3}$ & & & & \\
\hline $2^{-10}$ & $2^{-4}$ & 1.3872 & 1.2531 & 1.1841 & 1.1424 \\
\hline $2^{-10}$ & $2^{-5}$ & 1.5540 & 1.2676 & 1.1884 & 1.1425 \\
\hline $2^{-10}$ & $2^{-6}$ & 1.6878 & 1.4151 & 1.2607 & 1.1280 \\
\hline $2^{-10}$ & $2^{-7}$ & 1.7892 & 1.4580 & 1.1861 & 1.1961 \\
\hline
\end{tabular}

Table 8: The experimentally determined orders of convergence (EOC) at $t=1$ in Example 3.5 by using the shifted Diethelm's method 


\section{Conclusions}

As we have foreseen in the earlier discussion, we have constructed a reliable numerical scheme for the solution of equations of the form (1). We also have a convergence result and examples showing how the order is lost when the smoothness assumptions are violated. This gives an insight into how the method is likely to perform in practice. The use of numerical schemes usually has the effect of solving a slightly perturbed problem. Even if the original problem has solutions that satisfy the smoothness conditions, we may find that the perturbed problem has solutions that are not smooth. Thus the convergence order of the numerical solution will be reduced in the way we have seen in Example 3.3.

\section{References}

[1] J. CAO AND C. XU, A high order schema for the numerical solution of the fractional ordinary differential equations, J. Comp. Phys., 238(2013), 154-168.

[2] H. W. Choi, S. K. Chung And Y. J. Lee, Numerical solutions for space fractional dispersion equations with nonlinear source terms, Bull. Korean Math. Soc., 47 (2010), 1225-1234.

[3] W. Deng, Finite element method for the space and time fractional Fokker-Planck equation, SIAM J. Numer. Anal, 47(2008), 204-226.

[4] W. Deng and J. S. Hesthaven, Discontinuous Galerkin methods for fractional diffusion equations, ESAIM:M2AN, 47(2013), 1845-1864

[5] K. Diethelm, Generalised compound quadrature formulae for finite-part integrals, IMA J. Numer. Anal., 17(1997), 479493.

[6] K. Diethelm, An algorithm for the numerical solution of differential equations of fractional order, Elctronic Transactions on Numerical Analysis, 5(1997), 1-6.

[7] K Diethelm, J.M Ford N.J Ford and M Weilbeer, Pitfalls in fast numerical solvers for fractional differential equations, J. Comput. Appl. Math., 186 (2006), 482-503.

[8] Y. Dimitrov, Numerical approximations for fractional differential equations, arXiv: 1311.3935v1 [math. NA], 2013.

[9] V. J. ERvin AND J. P. Roop, Variational formulation for the stationary fractional advection dispersion equation, Numer. Methods Partial Differential Equations, 22(2006), 558-576.

[10] V. J. Ervin And J. P. Roop, Variational solution of fractional advection dispersion equations on bounded domains in $\mathbb{R}^{d}$, Numer. Methods Partial Differential Equations, 23(2007), 256-281.

[11] V. J. Ervin, N. Heuer And J. P. Roop, Numerical approximation of a time dependent nonlinear, space-fractional diffusion equation, SIAM J. Numer. Anal., 45(2007), 572-591.

[12] G.J. Fix AND J. P. RoOP, Least squares finite-element solution of a fractional order two-point boundary value problem, Comput. Math. Appl., 48(2004), 1017-1033.

[13] N. J. Ford, M. M. Rodrigues, J. XiAo And Y. Yan, Numerical analysis of a two-parameter fractional telegraph equation, Journal of Computational and Applied Mathematics, 249(2013), 95-106.

[14] N. J. Ford, J. XIAO AND Y. YAn, Stability of a numerical method for space-time-fractional telegraph equation, Computational Methods in Applied Mathematics, 12(2012), 1-16.

[15] M. Ilic, F. LIU, I. TURNer AND V. ANH, Numerical approximation of a fractional-in-space diffusion equation II: with nonhomogeneous boundary conditions, Frac. Calc. Appl. Anal., 9(2006), 333-349.

[16] X. J. Li AND C. J. Xu, A space-time spectral method for the time fractional diffusion equation, SIAM J. Numer. Anal., 47(2009), 2108-2131.

[17] X. J. Li AND C. J. XU , Existence and uniqueness of the weak solution of the space-time fractional diffusion equation and a spectral method approximation, Commun. Comput. Phys., 8(2010), 1016-1051.

[18] C. P. Li ANd F. Zeng, Finite difference methods for fractional differential equations, International Journal of Bifurcation and Chaos, 22(2012), 1230014.

[19] F. Liu, V. Anh And I. Turner, Numerical solution of space fractional Fokker-Planck equation, J. Comp. Appl. Math., 166(2004), 209-219.

[20] C. Lubich, Discretized fractional calculus, SIAM J. Math. Anal., 17(1986), 704-719

[21] V. E. Lynch, B. A. Carreras, D. Del-Castillo-Negrete, K. M. Ferreira-Mejias and H. R. Hicks , Numerical methods for the solution of partial differential equations of fractional order, J. Comput. Phys., 192(2003), 406-442.

[22] M. M. Meerschaert, D. A. Benson, and B. Baeumer, Multidimensional advection and fractional dispersion, Phys. Rev. E, 59 (1999), 5026-5028.

[23] M. M. MEerschaert and C. TADjeran, Finite difference approximations for fractional advection-dispersion flow equations, J. Comput. Appl. Math., 172 (2004), 65-77.

[24] M. M. Meerschaert and C. Tadjeran, Finite difference approximations for two-sided space-fractional partial differential equations, Applied Numerical Mathematics, 56(2006), 80-90.

[25] M. M. Meerschaert, H. Scheffler And C. Tadjeran, Finite difference methods for two-dimensional fractional dispersion equation, J. Comput. Phys., 211(2006), 249-261.

[26] Z. M. OdiBAt, Computational algorithms for computing the fractional derivatives of functions, Math. Comput. Simul. 79(2009), 2013-2020. 
[27] K. Oldham and J. Spanier, The Fractional Calculus, Academic Press, San Diego, 1974.

[28] I. Podlubny, Matrix approach to discrete fractional calculus, Fract. Calc. Appl. Anal., 3(2000), $359-386$.

[29] I. Podlubny, A. Chechkin, T. Skovranek, Y. Q. Chen and B. Vinagre, Matrix approach to discrete fractional calculus II: partial fractional differential equations, J. Comput. Phys., 228(2009), 3137-3153.

[30] E. SousA, How to approximate the fractional derivative of order $1<\alpha \leq 2$, The 4th IFAC Workshop Fractional Differentiation and Its Applications (Badajoz, Spain), Article no. FDA10-019, 6 pages.

[31] C. Tadjeran, M. M. Meerschaert And H. Scheffler, A second-order accurate numerical approximation for the fractional diffusion equation, J. Comput. Phys., 213(2006), 205-213.

[32] C. TAdjeran, M. M. Meerschaert, A second-order accurate numerical method for the two-dimensional fractional diffusion equation, J. Comput. Phys., 220(2007), 813-823.

[33] W. Tian, H. Zhou And W. Deng, A class of second order difference approximations for solving space fractional diffusion equations, Math. Comp. 84(2015), 1730-1727.

[34] Y. YAN, K. PAL AND N. J. FORD, Higher order numerical methods for solving fractional differential equations, BIT Numer Math, (2013), DOI 10.1007/s10543-013-0443-3.

[35] Q. YAng, F. LiU AND I. TURNer, Stability and convergence of an effective numerical method for the time-space fractional Fokker-Planck equation with a nonlinear source term, Int. J. Diff. Eqs. (2010), Article ID 464321, 22 pages. 